\title{
VERDI E IL SALOTTO MILANESE DI CLARA MAFFEI
}

\author{
FRANCA CELLA $(*)$
}

RiASSUNTO - Fra i salotti dell'Ottocento quello della Contessa Maffei ha un carattere speciale. Non solo per la durata singolare (52 anni) e la notorietà europea. E' un punto di ritrovo privato dove batte il cuore di Milano, si raccolgono aristocratici, intellettuali e artisti borghesi o popolani nobilitati dall'arte e dagli ideali, dove si muove la storia della città e dell'Italia. Il discorso coinvolge quattro personaggi: i due fondatori (nel 1834) Andrea e Clara Maffei, Giuseppe Verdi e sua moglie Giuseppina Verdi Strepponi, attraverso i loro reciproci documenti epistolari. Il carteggio comprende oltre 600 documenti, in parte noti e sparsamente pubblicati, in parte inediti (lettere di Clara conservate negli archivi di Sant'Agata); per continuità e numero di lettere il dialogo Verdi-Clara è nettamente dominante. Andrea Maffei (Molina di Ledro 1798- Milano 1885), è personaggio di spicco nella cultura italiana della prima metà dell'Ottocento: classicista di studi e prestigio letterario, conoscitore di poeti e teatro d'oltralpe diffonde in Italia il gusto internazionale con le tante traduzioni dal tedesco, sua seconda lingua (da Klopstock a tutto il teatro di Schiller) e di autori inglesi; amico di artisti si fa interprete attivo della consonanza fra letteratura, arte, musica. A Verdi, accolto nel salotto dal 1842, offre contatti formativi con letterati e artisti, e personalmente una scuola viva di gusto culturale e teatrale, orientandolo nella scelta dei soggetti, con la logica drammaturgica degli sbozzi, fino a comporgli il libretto de I Masnadieri, gli aggiustamenti al fantastico delle streghe nel Macbeth. Chiara Carrara Spinelli (Bergamo 1814-Milano 1886), è creatura incantevole, colta, lieve, vibrante di emozioni e ideali; al talento di relazioni del marito oppone quello di saper accogliere e ascoltare, aperta a tutti i tipi di soccorso spirituale, materiale e sociale. Il rapporto di Verdi con Clara è un'amicizia confidenziale, nobile, senza riserve. Cresce nella lontananza, poiché per 20 anni Verdi evita Milano, e rivedrà Clara solo nel '68 in occasione della visita a Manzoni. Ma le lettere di lei tracciano un filo continuo con amici antichi e nuovi del salotto, e proprio lei, che venera e frequenta Manzoni, sollecita il culto che già ne ha Verdi con notizie continue e lo spinge all'incontro. Quando è vicina, nei due soggiorni a Sant'Agata, cattura istantanee inedite dell'intimità verdiana. Per Clara Verdi rappresenta l'aspirazione suprema di arte ed etica, ed è orgogliosa d'essere partecipe delle sue scelte, rassicurata dal sostegno alle proprie inquietudini e fragilità. Con lei Verdi osa sfoghi dell'animo o definizioni sul vero, le affida delicate missioni di soccorso ad amici

(*) Critico musicale. Collaboratore dell'Istituto Nazionale di Studi Verdiani. 
o risponde ad altre che lei segnala, agevola rapporti epistolari tra Clara e Peppina che diventeranno intime amiche.

$* * *$

ABSTRACT - Among the 19th century literary salons an important role is played by that of the countess Clara Maffei, not only for its long duration (52 years) and its European reputation. It's a private meeting point, the heart of Milan where aristocrats, intellectuals and burgeois gather together. The four personalities involved are: the two founders Andrea and Clara Maffei, Giuseppe Verdi and his wife Giuseppina Verdi Strepponi with their correspondence. This epistolar exchange consists of more than 600 documents, partly wellknown and published and partly unpublished (Clara's letters conserved in Sant'Agata archives). Very important is the correspondence between Verdi and Clara. Andrea Maffei (Molina di Ledro 1798 - Milano 1885) stands out in the Italian culture of the mid nineteenth century: connoisseur of poets and theatre beyond the Alps, he spreads in Italy the International taste with many translations from german, his second language (from Klopstock to the theatre of Schiller) and English authors: he is friend of many artists and he represents actively the consonance among literature, art and music. He offers to Verdi, received in the salon in 1842, formative relationships with intellectuals and artists and personally a lively school of cultural and theatre taste, guiding him in the choice of subjects, he composes for Verdi the libretto of I Masnadieri and he makes the fantasy arrangements of the witches in Macbeth. Chiara Carrara Spinelli (Bergamo 1814 - Milano 1886) is a fascinating creature, cultured and quivering of emotions and ideals. She has a vocation in receiving and listening, she offered all kinds of spiritual, material and social assistance. The relationship between Clara and Verdi is based on a confidential, noble, without reserve friendship. A friendship that grows in the distance, as for 20 years Verdi stays away from Milan, and he will see Clara again in 1868 during the visit at Manzoni. But her letters marks a thread with old and new friends of the salon, and herself, who adores and spends time with Manzoni, stimulates the cult of Verdi for this personality with continuous news and pushes him to a meeting. During the two stays in Sant'Agata she captures intimate moments of Verdi's life. He represents for Clara the maximum ambition of art and ethics, and she is proud of sharing his choices, reassured about her apprehensions and weakness. Verdi opens his heart to Clara and facilitates the correspondence between Clara and Peppina who will become close friends.

Sto lavorando al Carteggio Verdi-Maffei in vista della pubblicazione per l'Istituto Nazionale di Studi Verdiani.

E' un corpus di oltre 600 documenti, in parte noti e sparsamente pubblicati, in parte inediti (le lettere di Clara a Verdi dall'Archivio di Sant'Agata), allargato con citazioni trasversali da altri carteggi dei protagonisti. La fase di lavoro attuale non mi permette ancora una sintesi di sistemazione scientifica. Ho preferito esporre l'interesse, la vitalità, la novità di contributi di questa storia di un'amicizia durata 44 anni. 


\section{PREMESSA}

Nella costellazione di salotti (politici, letterari, mondani) che accompagnano la storia dell'Ottocento quello della Contessa Maffei ha un carattere speciale.

Non solo per la durata singolare (52 anni) e per la notorietà europea, punto d'incontro di ospiti e salotti internazionali.

Il discorso che ci riguarda coinvolge quattro personaggi: i due fondatori Andrea e Clara Maffei, Giuseppe Verdi e sua moglie Giuseppina Verdi Strepponi, attraverso i reciproci documenti epistolari e la collaborazione del compositore col poeta Andrea. Le loro lettere si intrecciano ad altri carteggi dei protagonisti, importanti per verificare, precisare, completare un discorso che continuamente allarga i propri confini, raccoglie altre voci ed echi del salotto.

Chiara Carrara Spinelli (Bergamo 1814- Milano 1886), è nata in odor di poesia, figlia del Conte Giovanni Battista (poeta, autore di tragedie, pedagogo in casa Litta a Milano) e della Contessa Ottavia Gambara, con nonna materna poetessa, Chiara Trinali, e nonno morto perchè punto da una rosa. E' creatura incantevole, minuta, colta, lieve, vibrante di emozioni e ideali; fragile e instancabile ha l'arte innata dell'accoglienza, osserva e guida le conversazioni seduta dans son fauteuil, aperta a tutti i tipi di soccorso spirituale, materiale e sociale, animatrice tenace delle aspirazioni, scelte, battaglie, resistenza per la libertà e indipendenza d'Italia, protettrice dei giovani eroi come dei giovani artisti che affollano il suo salotto. Cura ammalati e feriti, sfida pericoli, raccoglie sussidi, e ogni domenica mattina, dopo Messa, fa visita ad Alessandro Manzoni, il suo Santo, premia gli amici con una fogliolina d'edera del suo balcone scivolata tra i fogli della lettera. A 18 anni, il 10 marzo 1832, sposa il poeta Andrea Maffei, letterato di spicco che ha 16 anni più di lei.

Andrea Maffei (Molina di Ledro 1798, Milano 1885) è un bell'uomo trentino: studi classici a Bologna poi a Monaco di Baviera, da uno zio consigliere della Casa regnante. Nel 1818 esordisce a Milano traducendo gli "Idilli" di Salomon Gessner e li dedica a Vincenzo Monti. Nel clima di fervore legato al "Conciliatore" e all'articolo di Madame de Staël Sulla maniera e la utilità delle traduzioni, in "Biblioteca Italiana", gennaio 1816, entra con l'arma vincente della lingua e cultura tedesca. Diffonderà il gusto internazionale per gli autori e il teatro d'oltralpe traducendo nel tempo Friedrich Gottlieb Klopstock 
(Messiade), Ladislaus Pyrker, Patriarca di Venezia (Tunisiade), Goethe (Faust), Byron (La fidanzata d'Abido, Caino, Il canto di Medora, Parisina, Il prigioniero di Chillon, Sardanapalo, Marin Faliero, I due Foscari), Thomas Moore, (Gli amori degli angeli, Canti Orientali, Le melodie, La luce dell'barem, Gli adoratori del fuoco, del 1859, dedicato a Verdi); Il teatro completo di Schiller, e anche Turandot di Carlo Gozzi imitata da Schiller, e Shakespeare imitato da Schiller (Macbeth, Otello, La Tempesta), Franz Grillparzer (Avola, Medea), John Milton (Il paradiso perduto), Struensee di Michael Beer, fratello di Meyerbeer. Professionista di una traduzione poetica che ricrea "a quel modo che una bella musica si ripete sopra un diverso strumento" (de Staël, cit.), coi suoi versi "ben periodati e cadenzati e sonanti" (Croce) ${ }^{1}$ piace a innovatori e a conservatori, acquista prestigio, relazioni, diventa consulente editoriale in varie città, Socio di Accademie (tra cui dal '28 quella dei Filodrammatici, dove Verdi si fa notare dirigendo La Creazione di Haydn, e dove Maffei nel '29 aveva presentato una Cantata con musica di Carlo Conti in onore di Monti, e dal '39 è membro della commissione dei drammi), amico di letterati antichi e di giovanissimi di cui promuove gli scritti, frequentatore di artisti (da Hayez, a Schiavoni, Vela, Bertini, Puttinati, Pompeo Marchesi, Conconi, Molteni..) cui suggerisce soggetti, illustra quadri e statue coi versi, commissiona, colleziona opere, convinto dell'armonico intreccio fra le arti sorelle; e non fu estraneo il suo influsso al gusto esotico (proposto attraverso Moore o Pyrker) ai soggetti della pittura storica o alla trasfigurazione allegorico politica delle immagini. Funzionario governativo prima a Venezia, Verona, quindi a Milano dov'è segretario del Tribunale d'Appello. Ha il dono naturale delle relazioni, dei contatti; perfino il Barone Torresani, direttore generale della Polizia, trentino e "amico leale fin dall'infanzia”, guarda con indulgenza il suo operare.

Nel 1834 Maffei fonda nella loro casa il salotto Maffei, dove invita aristocratici intellettuali e artisti in un clima di amicizia armoniosa regolato dalla moglie. Si riceve ogni giorno, dalle 15 alle 18, e dopo cena fino a mezzanotte. Si conversa, si promuovono idee e progetti, si legge, si fa musica, anche feste in onore di qualcuno o a fine anno.

Negli anni si avvicendano 4 sedi:

1 Benedetto Croce, La letteratura della nuova Italia. Saggi critici, Laterza, Bari, 1957, vol V, p. 194 
- dal 1834 via Monte di Pietà n. 1;

- dal 1842 Piazza Belgioioso n. 2, al $2^{\circ}$ piano di Palazzo Belgioioso, vicino alla casa del Manzoni.

E' il periodo letterario artistico elegante e internazionale pilotato dal Maffei. Tra i nomi Donizetti e Balzac, Liszt con Marie d'Agoult, Pompeo Belgioioso, Renato Borromeo, Felice Romani, Tommaso Grossi, Giulio Carcano, Francesco Hayez, talora Alessandro Manzoni, Luigi Toccagni, Ferdinando De Lugo, Angelo Fava, Giacinto Battaglia, Opprandino Arrivabene, Giovanni Prati, Carlo Cattaneo, Giuseppe Revere, Giovanni Rajberti, Antonio Gazzoletti, Jacopo Cabianca, Giuseppe Giusti, Felice Bellotti, Carlo Tenca e l'amico Alessandro Porro....

- dal 1846 (autunno) via del Giardino 46 (attuale Via Manzoni);

- dal 1850 fino all'86 via Bigli n. 21.

E' il periodo politico patriottico guidato da Carlo Tenca (Milano 1816- ivi 1883). Dal 15 giugno 1846 i coniugi Maffei si sono separati. Tenca si è legato a Clara, porta avanti con rigore l'ideale politico, fonda "Il Crepuscolo" che nasce in casa Maffei, ed uscirà ogni domenica per 10 anni, dal 1850 al '60. Nel salotto i più bei nomi delle Cinque Giornate, il filo conduttore con Cavour e il Piemonte, la propaganda e l'eroismo segreto di dieci anni di preparazione al '59. Fra i nomi Enrico Dandolo, Luciano Manara, Emilio Morosini, Carlo De Cristoforis, Agostino Bertani, Cesare Correnti, Anselmo Guerrieri Gonzaga, Cesare Giulini Dalla Porta...

Dagli anni '60 il salotto si riapre alla nuova cultura e società: Filippi e Faccio, Leone Fortis, Ruggero Bonghi, Camillo e Arrigo Boito, Emilio Praga, Gaetano Negri, Giulio Ricordi, Achille Torelli, Giovanni Verga, il compositore Gomes, ma anche la Stolz, la Pantaleoni, Vittoria Cima...

Verdi è accolto nel salotto dai primi mesi del 1842, dopo il successo di Nabucco. Introdotto da Andrea, che già doveva averlo conosciuto nel suo exploit del 1834 e 1835 all'Accademia dei Filodrammatici e successivamente al Casino dei Nobili. E' in atto- da parte di Anselm Gerhard ${ }^{2}$ lo studio del rapporto di patronage da parte

2 Anselm Gerhard,"Cortigiani, vil razza bramata!"Reti aristocratiche e fervori risorgimentali nella biografia del giovane Verdi(Prima parte), in "Acta Musicologica", vol. LXXXIV/ 1 (2012), pp.37-63; (Seconda parte), ibidem, vol.LXXXIV/2(2012), pp.199-223. 
dei circoli aristocratici di cui Maffei è il tramite culturale: dal Conte Renato Borromeo, Presidente dell'Accademia dei Filodrammatici, autore della Cantata commissionata a Verdi nel 1836, a Francesco Pasetti, membro della Commissione musica, a cui è dedicato Oberto. Il giovane Verdi è proteso ad afferrare meraviglie e novità di Milano e della sua cultura. Il rapporto col poeta Maffei è subito profondo, al nodo del teatro, del gusto attuale e raffinato, delle sue mitiche amicizie, delle novità di pittori, scultori, Esposizioni. Fino al 1849 c'è un filo di sollecitazione continua nella proposta di soggetti, nella sicurezza degli sbozzi preliminari, nell'attrazione per il Teatro di Schiller (5 opere su 26), oltre ai documentati aggiustamenti al libretto di Macbeth e a quello composto per Verdi de I Masnadieri. C'è in Verdi una naturalezza di consultazione e nel poeta l'offerta a prestare generosamente la propria collaborazione. C'è la passione visiva, il gusto che Verdi coltiverà sempre per Esposizioni e botteghe d'artisti; la passione per gli autografi dei grandi che Maffei negli anni gli spedisce assieme a disegni preziosi (Foscolo, Pindemonte, Giuseppe Niccolini...). C'è una forte solidarietà e comprensione delle intime cose. Quando nel giugno 1846 i coniugi Maffei si separano, per volontà di Clara, proprio Verdi e Giulio Carcano sono i testimoni dell'atto di separazione legale davanti al Notaio Tommaso Grossi. E Verdi, che ha capito la delicata situazione con riserbo e rispetto, cerca di proteggerli entrambi con vicinanza e conforto. Per mesi sta vicino all'amico, disperato perché nulla aveva intuito, prima alle acque di Recoaro e poi a Firenze coinvolgendolo nella preparazione del Macbeth. Il rapporto continuo con Maffei dura fino alla vigilia del '48, dal ' 49 gli eventi li separano: Verdi affonda le radici a Sant'Agata, Maffei prende dimora a Riva del Garda. A intermittenza le lettere segnalano contatti, scambi, suggerimenti importanti, ma la funzione di tramite attivo tra Verdi e la cultura milanese cosmopolita del primo Ottocento si è compiuta. Il compositore va a Parigi, si apre ad altri stimoli.

Il legame con Milano, con gli echi del salotto, si identifica con Clarina. Il rapporto di Verdi con lei è quello di un'amicizia senza confronti "Era mia amica da 44 anni!... Amica sincera e sicura! Non sapeva certo fare i versi di suo marito..ma invece che cuore! e che nobiltà di carattere! e che elevatezza di sentimenti!" (a Giuseppe Piroli, Sant'Agata 23 luglio 1886). "Aveva tante qualità, ma la qualità sovrana era la bontà e l'indulgenza! Impossibile averne di più! Amica a tutta prova; e con Lei si era sicuri, non vi era bisogno di guardarsi alle spal- 
le!..Povera Clarina! Io non la dimenticherò così presto!!” (a Giuseppina Negroni, Sant'Agata, 23 luglio 1986). Depone l'innata diffidenza Verdi, trova il tempo per rispondere ai frequenti messaggi e lettere di questa creatura trasparente, straordinariamente dotata delle facoltà di sentimento, preveggenza, cuore, immaginazione, entusiasmo, aspirazione alla bellezza unita alla bontà, alla fede che dà slancio alla sua spiritualità. Per Clara Verdi personifica l'aspirazione suprema di arte ed etica costruttive di presente e futuro, l'identità di mente e cuore, di vigore e delicatezza. Lo venera ma è orgogliosa d'esser testimone e partecipe delle sue scelte, intuitiva e vigile come una sorella: "el Clarinett che dice sempre il vero" come si autodefinisce. Con sicurezza smentisce la diceria del Verdi orso, contraddice l'editore Escudier che ha appena pubblicato un ritrattino di Verdi uomo e intanto ci fa vedere Verdi giovane nel suo salotto: "vi trovai nell'intimità quale veramente lo siete, ma esagerò la sauvagerie nei salons, nel mio almeno foste sempre un serio ma cortese signore, e non mi spaventaste mai coll'aspetto d'un orso" (Milano, 17 novembre 1863). Sa prevedere le intenzioni del Maestro, e non lo contraddice mai: "ma io rispetto sempre i vostri $S i$; i vostri No anche senza giustificarli; la stima immensa che ho anche del vostro criterio mi fa veramente sottomettere a quanto credete fare o non fare." (Milano, 28 aprile 1871). Su un solo argomento punta i piedi con fermezza, quando Verdi contrariato, deluso, stanco si lascia scappare la tentazione di non far nulla. "Parlatemi dei vostri progetti (...) colla ricca, freschissima, dotta, alta facoltà che avete, è un dovere lo scrivere, il fare ancora qualche lavoro che esalti e sia di lezione a tutti." (Milano, 23 giugno 1876). Le parole forti di una voce solitamente dolcissima scuotono il Maestro, che spiega, si sfoga e ci pensa su: Cosa conto fare?...E' un dovere lo scrivere ancora!! Voi dite! Ma nò: e conto proprio di far niente. D'altronde a che servirebbe? (Sant'Agata, 1 luglio 1876).

Verdi comprende l'autenticità di Clarina, del suo sentire vibrante ai limiti della resistenza nervosa e le dà appoggio, confidenza, fiducia. $\mathrm{Ma}$ anche riceve.

Nel carteggio parla di tutto, spesso per accenni, sicuro d'essere intuito; sussurra sfoghi, rimugina impulsi polemici e tentazioni segrete dei suoi pensieri. Colpisce la sintonia di partecipazione politica nei loro incipit che si incrociano fra esaltazione e immediata delusione del '59, sull'angoscia per la Francia ferita nel '70. Clara dà notizie sempre aggiornate su amici e amiche comuni, racconta la vita del salotto, i suoi 
nuovi e tanti "intimi", come lei chiama gli ospiti. Protegge giovani di talento, come Boito e Faccio, "i mes car fioeu", che le suonano opere intere al pianoforte, e per loro chiede consigli al Maestro, e dovrà mediare con diplomazia posizioni divergenti e ricucire con pazienza l'incauta offesa che Boito gli arreca nel '63, con l'ode goliardica All'arte italiana. Di musica è adoratrice, ma lascia il campo agli addetti ai lavori; invece stimola Verdi sul teatro di prosa di cui lui è assiduo frequentatore e lei aggiornatissima, e provoca riflessioni e definizioni decisive come Inventare il Vero a proposito di Colore del Tempo di Achille Torelli. ${ }^{3}$

Sensibilissima ai bisogni sociali, alle difficoltà materiali che possono colpire gli amici, si adopera a soccorrerli, a coinvolgere la sensibilità di Verdi in aiuti generosi, spesso anonimi per delicatezza; a sua volta il Maestro le affida situazioni difficili come la grave malattia e il ricovero dell'amico Piave. Per l'Album che ha promosso per lui a scopo benefico Verdi mette in azione la funzione sociale e mediatica del salotto: “Battete dumque un po' la gran Cassa, e fate che i vostri adoratori, e le vostre adoratrici, e gli adoratori delle vostre adoratrici non solo comprino l'Album, ma ne dicano tutto il bene possibile, e lo facciano diventare alla moda." (Sant'Agata, 20 ottobre 1876).

Per anni Verdi, lontano da Milano, non fa cenno del suo rapporto con Giuseppina Strepponi. L'ambiente musicale sa, cantanti che frequentano Parigi, dove lei vive dal '46 e insegna canto, segnalano d'aver incontrato Verdi da lei. Ma il compositore tace. Nell'aprile '56 troviamo il primo ringraziamento anche a nome di Peppina per un "biglietto graziosissimo" di Clara; la strategia d'accoglienza cresce: "La Peppina è sensibilissima alle parole affettuose che le piacque dire a suo riguardo. Essa le vuol bene come se la conoscesse personalmente, e si terrà contenta ed onorata se vorrà dargli un modestissimo posto fra le persone che la amano e la stimano... e sono pur molte" (Busseto, 25 marzo 1857). Ma solo nel maggio '67, e per iniziativa della moglie di Verdi che si presenta sola e inattesa in via Bigli, la foto del Maestro come biglietto da visita, nascerà un'amicizia sincera e palpitante.

Un carteggio così vivo, nasconde una storia di lontananze. Perché dal 31 maggio del '48 Verdi lascia definitivamente Milano, e non vi tor-

3 Lettera di Giuseppe Verdi, Sant'Agata 14 novembre 1869, a Clara Maffei, Milano. 
nerà fino al giugno del '68 per l'incontro privato con Manzoni. Per vent'anni non vede Clara, che viene invitata per un primo soggiorno a Sant'Agata nel maggio '68, e poi ancora nel '69, sfinita per esser corsa a Firenze in aiuto al marito Andrea, gravemente ammalato. Clara s'immerge nella pace della campagna, nei ritmi quotidiani della casa, e il suo sguardo cattura istantanee dell'intimità verdiana. Le fissa nelle lettere di gratitudine, di memoria che rivive. La passeggiata in giardino alle sette, Verdi che coglie le rose destinate a Manzoni; Verdi che "non sarebbe detto taciturno, parco almeno di parola, se alcuno avesse potuto udire gli animati e prolungati nostri colloqui" (Milano, 26 maggio 1868). Il Maestro spiato nella concentrazione del comporre: "mi pare vederlo là fra il cembalo e quel comodo sofà provare e meditare e chiamarti poi..." (a Giuseppina Verdi, Milano 21 novembre 1870). Immaginato, sulla continuità di quell'immagine, nel fervore di scrivere la Messa di Requiem per onorare Manzoni. "Io scorgo col pensiero Verdi nella sua stanza, ora al piano, ora sdrajato sul Canapé vicino, a pensare e scrivere la Messa pel nostro Santo" (a Giuseppina Verdi, Milano,18 dicembre 1873). E' forse questo il dono più alto dell'amicizia di Clara: aver coltivato l'emozione e devozione che Verdi sentiva già per Manzoni, per il suo libro "vero", vincendo la titubanza dell'artista ad avvicinarlo, reggendolo all'incontro che, quando avvenne, gli parve un sogno. Da quel momento Manzoni, le sue parole, incontri, eventi, diventano un filo continuo delle lettere di Clara, in un crescendo di vicinanza, di sgomento quando la mente eletta dello scrittore comincia a smarrirsi, quando rifiuta quasi delirando la morte del figlio. Con parole essenziali e dolenti Clarina trasmette situazioni, voci che "schiantano il cuore", e avranno parte negli spazi di sgomento e vertigine della partitura verdiana.

Dal '69 Verdi è tornato a frequentare Milano, con periodici impegni alla Scala. Alloggia al Grand Hotel et de Milan, vis à vis alle finestre di Clarina. Rivede con piacere vecchi amici d'un tempo, in ore propizie, quando gli ospiti ancora non affollano via Bigli. Ma è stretto dal ritmo del teatro; salva il tempo per visite scambievoli, anche quotidiane, con l'amica fedele. Lei, più fragile e afflitta da dispiaceri, si profonde in gratitudine e consolazione. Verdi, con delicatezza, le rinnova "il piacere di trattenermi con Voi" (Sant'Agata, 19 giugno 1878); la rassicura "Son io che devo ringraziarvi, ed esservi riconoscente dell'affezione sincera che conservate sempre per me, e dell'accoglienza che mi dimostrate ogni volta che ho il piacere di vedervi a Milano" (Genova, 29 gennaio 1884). Quasi a fine corsa, morto già Tenca ed ora anche Maffei, Clara ringrazia Verdi 
per la forza morale e di amicizia con cui l'ha sostenuta nel tormentato cammino dalla separazione alla ritrovata amicizia e responsabilità verso il marito: "Non sono ringraziamenti, ma benedizioni che il povero mio animo vi manda per tutto il bene infinito, indicibile, che mi prodigaste in questi tristissimi giorni, mi consolaste col vostro prezioso affetto, del quale sono commossa sempre ed orgogliosa, mi reggeste col tranquillizzarmi sempre più la coscienza, e questo è il supremo dei beni! E' dolce il perdonare, è duro, è terribile punizione il riconoscere d'aver avuto torto, e fatto ciò che non si doveva!" (Milano, 8 dicembre 1885). Verdi ascolta, e si sente capito "Son proprio molto grato per la lettera che m'avete scritto," (Genova, 11 dicembre 1885). 